\begin{tabular}{c}
\hline \hline Juntendo \\
Research Profiles \\
\hline \hline Juntendo Medical Journal \\
2015. 61 (3), 318-319
\end{tabular}

\title{
Department of Metabolism and Endocrinology
}

\section{Members}

Professor \& Chairperson: Hirotaka Watada

Senior Associate Professor: Yoshio Fujitani

Associate Professor: Chie Ohmura, Akio Kanazawa,

Tomoaki Shimizu, Takeshi Ogihara, Yoshifumi

Tamura, Fuki Ikeda, Takeshi Miyatsuka,

Toyoyoshi Uchida, Tomoya Mita

Assistant Professor: Koji Komiya, Hiromasa Goto, Kageumi Takeno

\section{Research}

\section{Background}

Over seven million Japanese are estimated to have diabetes mellitus, a serious and costly disease. Diabetes causes several major complications, such as blindness, kidney failure, leg and foot amputations, and cardiovascular diseases. People with diabetes have a decreased ability to secrete sufficient insulin, a hormone that allows glucose to enter cells and be converted to energy. If diabetes is not controlled well, excess glucose remains in the blood and damages a lot of organs through vessels throughout the body. The long-term goal of our research is to develop better care for those with diabetes. To achieve this, we are focusing on the following projects:
1. The role of autophagy in $\beta$-cell homeostasis

Common features of type 2 diabetes mellitus are progressively decreased pancreatic $\beta$-cell function and $\beta$-cell mass, resulting in insufficient insulin secretion. We have shown that dysfunction of cellular autophagy occurs in islets of diabetic patients as well as mice under metabolic stress, such as high-fat-fed mice and $\mathrm{db} / \mathrm{db}$ mice, and that autophagy-deficient mutant mice exhibit impaired glucose tolerance, partly due to the lack of a compensatory increase in $\beta$-cell mass ${ }^{1)}$. In addition, we have recently found that the forced expression of human IAPP, which is thought to cause $\beta$-cell failure in diabetic patients, caused deterioration of glucose tolerance in mice with a $\beta$-cell-specific autophagy defect, indicating that increased autophagy may enhance the toxic potential of hIAPP in diabetic patients ${ }^{2}$. Now, we are exploring further molecular mechanisms of how autophagic dysfunction is induced in $\beta$ cells under conditions of metabolic stress, and what role autophagy has in regulating $\beta$-cell survival and/or replication.

\section{Regulation of glucose profiles by zinc signals}

Zinc is an essential nutrient for living organisms because its deficiency causes growth retardation, immunodeficiency, hypogonadism, and neuronal and

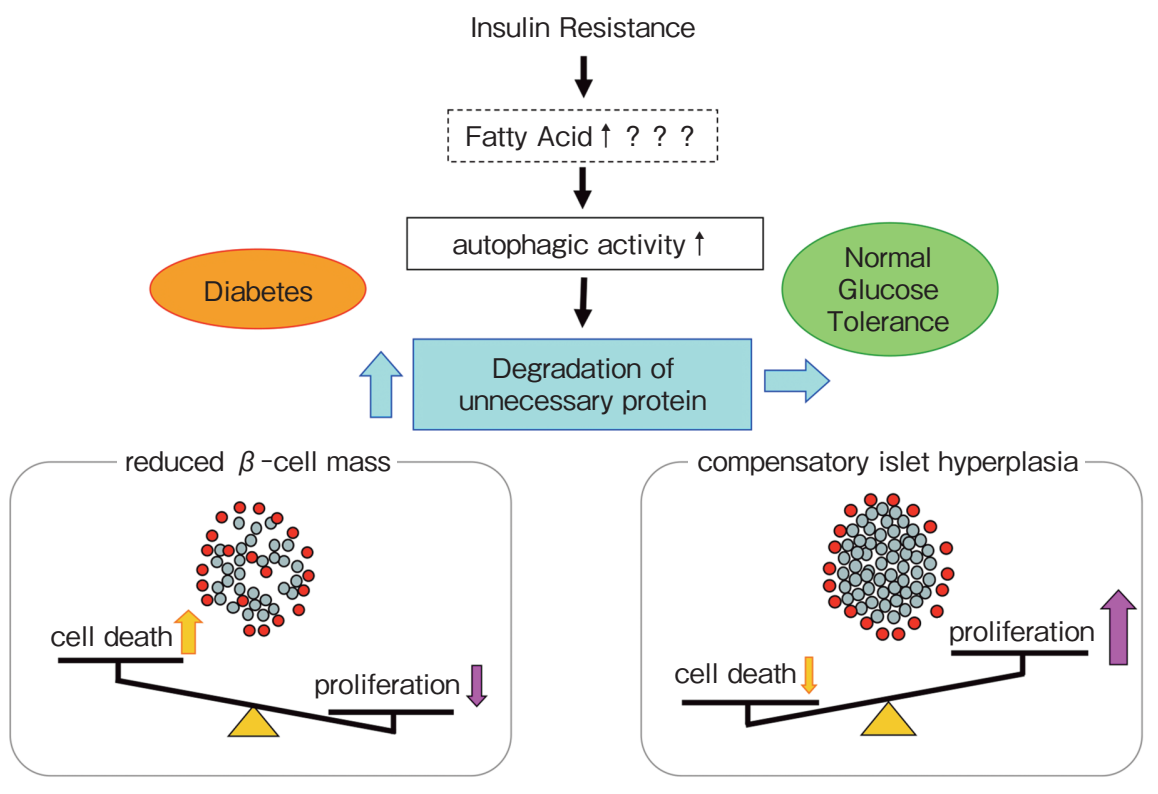

Figure-1 Role of $\beta$ cell autophagy in insulin resistance 
sensory dysfunctions. Intracellular zinc homeostasis is controlled via coordinated regulation of zinc influx and efflux, where zinc transporters have essential roles. Recent genome-wide association studies (GWAS) have demonstrated that common variants of SLC30A8 increase susceptibility to type 2 diabetes. SLC30A8 encodes zinc transporter-8 (ZnT8), which delivers zinc from the cytoplasm into insulin granules. In order to uncover the roles of $\mathrm{ZnT} 8$ and its pathological implications, we have generated $\beta$-cell-specific Slc30a8deficient mice. We demonstrated that zinc $\mathrm{co}^{-}$secreted with insulin suppressed hepatic insulin clearance ${ }^{3)}$. Our study highlighted a novel role of zinc-mediated intraorgan communication, referred to as "zinc flow", between the pancreas and the liver.

We are currently investigating further molecular factors underlying the role of zinc in pathological conditions of lifestyle-related diseases.

\section{Understanding the progression of atherosclerosis in diabetic patients}

Patients with type 2 diabetes mellitus are at high risk of developing cardiovascular diseases. However, it largely remains unknown which risk factors in diabetic conditions contribute to the progression of atherosclerosis because diabetic patients have many risk factors, such as hyperglycemia accompanied by insulin resistance, hyperinsulinemia, hypertension, and hyperlipidemia.

In order to quantify the adhesion of monocytes to the endothelium, which is one of the earliest events in naturally occurring experimental animal models of atherosclerosis in vivo, we have established a new en face method for optimal observation of endothelial surface (NEMOes) ${ }^{4)}$. This method allows us to observe the entire surface of the endothelium with a clear focused image, and thus to quantify the number of monocytes adhering to every region of rodent thoracic aorta. Using this NEMOes method, we are uncovering how each risk factor contributes to the progression of atherosclerosis, which would lead to new therapeutic approaches for preventing and curing cardiovascular diseases in patients with type 2 diabetes.

\section{Investigating mechanisms of insulin resistance in muscle and liver}

In most Asian countries, type 2 diabetes can easily develop in subjects with normal body mass index (BMI) $\left(<25 \mathrm{~kg} / \mathrm{m}^{2}\right)$, in contrast to the case in European countries and the USA, although the mechanisms that induce metabolic disorders in normal-weight subjects are not fully understood.

It has been shown that ectopic fat accumulation in muscle and liver induces insulin resistance in these organs, independent of obesity. We have measured intramyocellular lipid (IMCL) and intrahepatic lipid (IHL), using proton magnetic resonance spectroscopy $(1 \mathrm{H}-\mathrm{MRS})$, in order to investigate the role of ectopic fat accumulation in insulin resistance, and found that a short period of calorie restriction and exercise therapy decreased IHL and IMCL, respectively ${ }^{5)}$, suggesting that dietary and exercise intervention in metabolic diseases may directly decrease ectopic fat and improve metabolic states in liver and muscle, independent of body weight reduction.

On the basis of these findings, we hypothesized that diet and physical activity directly regulate intracellular lipid accumulation and insulin sensitivity in muscle and liver independently of obesity. To test this hypothesis, we have recently evaluated tissue-specific insulin resistance in muscle and liver of nonobese diabetic patients by using a euglycemic hyperinsulinemic clamp with a glucose tracer, searching for determinants of insulin resistance, such as ectopic fat and lifestyle factors. We also generated a mouse model of physical inactivity and investigated how physical inactivity regulates IMCL and insulin sensitivity in muscle. This approach will help us to understand the pathophysiology of metabolic disorders in non-obese diabetic subjects and bring new insight for treatment beyond the idea of body weight reduction.

\section{References}

1) Ebato C, et al: Autophagy is important in islet homeostasis and compensatory increase of beta cell mass in response to high-fat diet. Cell Metab, 2008; 8: 325-332.

2) Shigihara N, et al: Human IAPP-induced pancreatic $\beta$ cell toxicity and its regulation by autophagy. J Clin Invest, 2014; 124: 3634-3644.

3) Tamaki M, et al: The diabetes-susceptible gene SLC30A8/ZnT8 regulates hepatic insulin clearance. J Clin Invest, 2013; 123: 4513-4524.

4) Ervinna N, et al: Anagliptin, a DPP-4 inhibitor, suppresses proliferation of vascular smooth muscles and monocyte inflammatory reaction and attenuates atherosclerosis in male apo E-deficient mice. Endocrinology, 2013; 154: 1260-1270.

5) Tamura Y, et al: Effects of diet and exercise on muscle and liver intracellular lipid contents and insulin sensitivity in type 2 diabetic patients. J Clin Endocrinol Metab, 2005; 90: 3191-3196. 